\title{
Ulam stability to a toppled systems of nonlinear implicit fractional order boundary value problem
}

\author{
Zeeshan Ali ${ }^{*}$, Akbar Zada $^{1}$ and Kamal Shah ${ }^{2}$
}

\section{"Correspondence:}

zeeshanmaths1@gmail.com

'Department of Mathematics,

University of Peshawar, Peshawar,

Pakistan

Full list of author information is

available at the end of the article

\section{空 Springer}

\begin{abstract}
In this manuscript, we give some sufficient conditions for existence, uniqueness and various kinds of Ulam stability for a toppled system of fractional order boundary value problems involving the Riemann-Liouville fractional derivative. Applying the Banach contraction principle and the Leray-Schauder result of cone type, uniqueness and existence results are proved for the proposed toppled system. Stability is investigated by using the classical technique of nonlinear functional analysis. The results obtained are well illustrated with the aid of an example.
\end{abstract}

MSC: 34A08; 34B15; 34B27

Keywords: Riemann-Liouville derivative of fractional order; Implicit toppled system; Existence theory; Green function; Ulam stability

\section{Introduction}

FODEs have recently been addressed by many researchers for a variety of problems. The aforesaid equations arise in many engineering and scientific disciplines as the mathematical modeling of processes and systems in the fields of signal and image processing, control theory, physics, blood flow phenomena, polymer rheology, electrodynamics of complex medium, chemistry, aerodynamics, economics, biophysics, etc. For details, see $[18,23,24,29-33]$ and the references cited therein. FODEs also serve as an excellent tool for the description of hereditary properties of different processes and materials. Moreover, one has found that the aforesaid model real world problems are more accurate than differential equations of integer order. In consequence, the subject of the foregoing equations are receiving great attention from the researchers. However, the theory of boundary value problems for nonlinear FODEs is still in the initial stages and many aspects of this theory need to be explored.

The research area which is most preferable in the field of FODEs and got incredibly much attention from the researchers is devoted to the existence theory of solutions. Many researchers have established some interesting results of the existence of solutions to boundary value problems for FODEs by applying different fixed point approaches. For a detailed study, see $[1,13,36,37]$ and the references cited therein. On the other hand, the investigation of toppled systems of the differential equations is also very significant because systems

(c) The Author(s) 2018. This article is distributed under the terms of the Creative Commons Attribution 4.0 International License (http://creativecommons.org/licenses/by/4.0/), which permits unrestricted use, distribution, and reproduction in any medium, provided you give appropriate credit to the original author(s) and the source, provide a link to the Creative Commons license, and indicate if changes were made. 
of this kind appear in various problems of applied nature. For details and examples, the reader may refer to $[2,10,15,17,25]$ and the references cited therein.

Another area of research, which has received considerable attention from the researchers is stability analysis of the differential equations in the sense of Ulam and their different kinds. The aforesaid stability was introduced by Ulam [40], in 1940. A significant breakthrough came in the following year, when Hyers [19] gave a partial answer to Ulam's problem. In addition to the aforesaid investigations, many researchers have studied the Ulam stability for differential equations of different orders; see [20, 21, 27, 28, 34, 47, 48] and the references cited therein. In last few years, authors [41] studied various kinds of Ulam stability for impulsive ordinary differential equations. In [46], authors studied various kinds of the aforesaid stability for impulsive FODEs. In [43], authors studied the Ulam stability for linear fractional equations. The above-mentioned stabilities [14] for FODEs are quite significant in realistic problems, biology, economics and numerical analysis. For details and examples, see $[4,5,7,11,12,26,42,44,45]$ and the references cited therein.

Ali et al. [8], investigated existence theory and different kinds of stability in the sense of Ulam for the following implicit fractional differential equations:

$$
\left\{\begin{array}{l}
D^{p} u(t)-\alpha\left(t, u(t), D^{p} u(t)\right)=0 \\
\left.D^{p-2} u(t)\right|_{t=0^{+}}=\left.\gamma_{1} D^{p-2} u(t)\right|_{t=T^{-}}, \\
\left.D^{p-1} u(t)\right|_{t=0^{+}}=\left.\beta_{1} D^{p-1} u(t)\right|_{t=T^{-}},
\end{array}\right.
$$

where $\mathrm{t} \in \mathrm{J}=[0, \mathrm{~T}]$ with $\mathrm{T}>0,1<\mathrm{p} \leq 2, \beta_{1}, \gamma_{1} \neq 1$ and $\alpha: \mathrm{J} \times \mathbb{R} \times \mathbb{R} \rightarrow \mathbb{R}$. Nowadays, researchers are devoting their work to the investigation of different kinds of stability in the sense of Ulam for toppled system of FODEs. For details, see [6, 22, 38, 39]. Recently, Ali et al. [9] investigated existence theory and different kinds of stability in the sense of Ulam for the following implicit toppled system:

$$
\left\{\begin{array}{l}
{ }^{c} D^{p} u(t)-\alpha\left(t, y(t),{ }^{c} D^{p} u(t)\right)=0 \\
{ }^{c} D^{q} y(t)-\chi\left(t, u(t),{ }^{c} D^{q} y(t)\right)=0 \\
\left.u^{\prime}(t)\right|_{t=0}=\left.u^{\prime \prime}(t)\right|_{t=0}=0,\left.\quad u(t)\right|_{t=1}=\lambda u(\eta) \\
\left.y^{\prime}(t)\right|_{t=0}=\left.y^{\prime \prime}(t)\right|_{t=0}=0,\left.\quad y(t)\right|_{t=1}=\lambda y(\eta)
\end{array}\right.
$$

where $\mathrm{t} \in \mathrm{J}=[0,1], 2<\mathrm{p}, \mathrm{q} \leq 3,0<\lambda, \eta<1$ and $\alpha, \chi: \mathrm{J} \times \mathbb{R} \times \mathbb{R} \rightarrow \mathbb{R}$ are continuous functions.

Influenced from the aforesaid discussion. In this manuscript, our objective to study the existence, uniqueness and various kinds of stability in the sense of Ulam for the given toppled system

$$
\left\{\begin{array}{l}
D^{p} u(t)-\alpha\left(t, y(t), D^{p} u(t)\right)=0 ; \\
D^{q} y(t)-\chi\left(t, u(t), D^{q} y(t)\right)=0 ; \\
\left.D^{p-2} u(t)\right|_{t=0^{+}}=\left.\gamma_{1} D^{p-2} u(t)\right|_{t=T^{-}},\left.\quad D^{p-1} u(t)\right|_{t=0^{+}}=\left.\beta_{1} D^{p-1} u(t)\right|_{t=T^{-}}, \\
\left.D^{q-2} y(t)\right|_{t=0^{+}}=\left.\gamma_{2} D^{q-2} y(t)\right|_{t=T^{-}},\left.\quad D^{q-1} y(t)\right|_{t=0^{+}}=\left.\beta_{2} D^{q-1} y(t)\right|_{t=T^{-}},
\end{array}\right.
$$

where $\mathrm{t} \in \mathrm{J}=[0, \mathrm{~T}], \mathrm{T}>0,1<\mathrm{p}, \mathrm{q} \leq 2$ and $\beta_{1}, \beta_{2}, \gamma_{1}, \gamma_{2} \neq 1 . \mathrm{D}^{\mathrm{p}}, \mathrm{D}^{\mathrm{q}}$ are Riemann-Liouville derivatives of fractional order and $\alpha, \chi: J \times \mathbb{R} \times \mathbb{R} \rightarrow \mathbb{R}$ are continuous functions. 
The manuscript is organized as follows. In Sect. 2, we present some basic materials needed to prove our main results. In Sect. 3, we set up some appropriate conditions for the existence and uniqueness of solutions to the proposed system (1.1) by applying some standard fixed point principles. In Sect. 4, we built up conditions for stability in the sense of Ulam to the solution of the proposed system (1.1). An example to illustrate our results is presented in Sect. 5 .

\section{Background materials}

In this section, we recall some definitions and preliminary results, which will be used throughout the manuscript.

Definition 2.1 ([3]) The Riemann-Liouville fractional integral of order $p>0$ for a function $u: \mathbb{R}^{+} \rightarrow \mathbb{R}$ is defined as

$$
\mathrm{I}^{\mathrm{p}} \mathrm{u}(\mathrm{t})=\frac{1}{\Gamma(\mathrm{p})} \int_{0}^{\mathrm{t}} \frac{\mathrm{u}(\mathrm{s})}{(\mathrm{t}-\mathrm{s})^{1-\mathrm{p}}} \mathrm{ds}
$$

provided that the integral exists.

Definition 2.2 For a function $u: \mathbb{R}^{+} \rightarrow \mathbb{R}$, the Riemann-Liouville derivative of fractional order $p>0, n=[p]+1$, is defined as

$$
D^{p} u(t)=\frac{1}{\Gamma(n-p)}\left(\frac{d}{d t}\right)^{n} \int_{0}^{t} \frac{u(s)}{(t-s)^{p-n+1}} d s,
$$

provided that integral on the right side exists. [p] denotes the integer part of the real number $p$. For more properties, the reader may refer to [3].

Lemma 2.1 The solution of the differential equation

$$
D^{p} u(t)=\varrho(t), \quad p>0,
$$

is given as

$$
I^{p} D^{p} u(t)=I^{p} \varrho(t)+k_{1} t^{p-1}+k_{2} t^{p-2}+\cdots+k_{n-1} t^{p-n-1}+k_{n} t^{p-n}
$$

where $\mathrm{n}=[\mathrm{p}]+1$ and $\mathrm{k}_{\mathrm{i}}, \mathrm{i}=1,2, \ldots, \mathrm{n}$, are real constants.

Lemma 2.2 Suppose $\mathrm{E}=\{\mathrm{u}(\mathrm{t}) \mid \mathrm{u} \in \mathrm{C}(\mathrm{J})\}$ is a Banach space endowed with a norm defined as $\|\mathrm{u}\|_{\mathrm{E}}=\max _{\mathrm{t} \in \mathrm{J}}|\mathrm{u}(\mathrm{t})|$. Similarly, the norm defined on the product space is $\|(\mathrm{u}, \mathrm{y})\|_{\mathrm{E} \times \mathrm{E}}=$ $\|\mathrm{u}\|_{\mathrm{E}}+\|\mathrm{y}\|_{\mathrm{E}}$. Obviously $\left(\mathrm{E} \times \mathrm{E},\|(\mathrm{u}, \mathrm{y})\|_{\mathrm{E} \times \mathrm{E}}\right)$ is a Banach space. Also, the cone $\check{\mathbb{C}} \subset \mathrm{E} \times \mathrm{E}$ is defined as

$$
\check{\mathbb{C}}=\{(\mathrm{u}, \mathrm{y}) \in \mathrm{E} \times \mathrm{E}: \mathrm{u}(\mathrm{t}) \geq 0, \mathrm{y}(\mathrm{t}) \geq 0\} .
$$

Theorem 2.1 ([16]) Suppose $\mathrm{E}$ a Banach space contains a cone $\check{\mathbb{C}}$ and if $\mathfrak{D} \subset \check{C}$ with $0 \in \mathfrak{D}$ is relatively open set. Let the operator $\mathrm{T}: \mathfrak{D} \rightarrow \mathfrak{D}$ be completely continuous. Then one of the following conditions exists: 
(1) there is $\mathrm{u} \in \partial \mathfrak{D}$ and $\delta \in(0,1)$ such that $\mathrm{u}=\delta \mathrm{Tu}$;

(2) Thas a fixed point in $\mathfrak{D}$.

Definition 2.3 ([35]) The proposed system (1.1) is Ulam-Hyers stable, if there are $C_{\mathrm{p}, \mathrm{q}}=$ $\left(C_{\mathrm{p}}, C_{\mathrm{q}}\right)>0$ such that, for some $\epsilon=\left(\epsilon_{\mathrm{p}}, \epsilon_{\mathrm{q}}\right)>0$ and for each $\mathrm{t} \in \mathrm{J}$ and solution $(\mathrm{u}, \mathrm{y}) \in \mathrm{E} \times \mathrm{E}$ of the following:

$$
\left\{\begin{array}{l}
\left|D^{\mathrm{p}} \mathrm{u}(\mathrm{t})-\alpha\left(\mathrm{t}, \mathrm{y}(\mathrm{t}), \mathrm{D}^{\mathrm{p}} \mathrm{u}(\mathrm{t})\right)\right| \leq \epsilon_{\mathrm{p}} \\
\left|\mathrm{D}^{\mathrm{q}} \mathrm{y}(\mathrm{t})-\chi\left(\mathrm{t}, \mathrm{u}(\mathrm{t}), \mathrm{D}^{\mathrm{q}} \mathrm{y}(\mathrm{t})\right)\right| \leq \epsilon_{\mathrm{q}} .
\end{array}\right.
$$

There is a unique solution $(\omega, \vartheta) \in \mathrm{E} \times \mathrm{E}$ with

$$
|(\mathrm{u}, \mathrm{y})(\mathrm{t})-(\omega, \vartheta)(\mathrm{t})| \leq C_{\mathrm{p}, \mathrm{q}} \epsilon
$$

Definition 2.4 ([35]) The proposed system (1.1) is generalized Ulam-Hyers stable, if there is $\Theta_{p, q} \in C\left(\mathbb{R}^{+}, \mathbb{R}^{+}\right)$with $\Theta_{p, q}(0)=0$, such that, for each $t \in J$ and solution $(u, y) \in E \times E$ of (2.1), there is a unique solution $(\vartheta, \omega) \in E \times E$ of (1.1), which satisfies

$$
|(\mathrm{u}, \mathrm{y})(\mathrm{t})-(\omega, \vartheta)(\mathrm{t})| \leq \Theta_{\mathrm{p}, \mathrm{q}}(\epsilon)
$$

Definition 2.5 ([35]) The proposed system (1.1) is Ulam-Hyers-Rassias stable with respect to $\Phi_{\mathrm{p}, \mathrm{q}}=\left(\Phi_{\mathrm{p}}, \Phi_{\mathrm{q}}\right) \in \mathrm{C}(\mathrm{J}, \mathbb{R})$, if there are constants $C_{\Phi_{\mathrm{p}}, \Phi_{\mathrm{q}}}=\left(C_{\Phi_{\mathrm{p}}}, C_{\Phi_{\mathrm{q}}}\right)>0$ such that, for some $\epsilon=\left(\epsilon_{\mathrm{p}}, \epsilon_{\mathrm{q}}\right)>0$ and for each $\mathrm{t} \in \mathrm{J}$ and solution $(\mathrm{u}, \mathrm{y}) \in \mathrm{E} \times \mathrm{E}$ of the following:

$$
\left\{\begin{array}{l}
\left|\mathrm{D}^{\mathrm{p}} \mathrm{u}(\mathrm{t})-\alpha\left(\mathrm{t}, \mathrm{y}(\mathrm{t}), \mathrm{D}^{\mathrm{p}} \mathrm{u}(\mathrm{t})\right)\right| \leq \Phi_{\mathrm{p}}(\mathrm{t}) \epsilon_{\mathrm{p}} \\
\left|\mathrm{D}^{\mathrm{q}} \mathrm{y}(\mathrm{t})-\chi\left(\mathrm{t}, \mathrm{u}(\mathrm{t}), \mathrm{D}^{\mathrm{q}} \mathrm{y}(\mathrm{t})\right)\right| \leq \Phi_{\mathrm{q}}(\mathrm{t}) \epsilon_{\mathrm{q}}
\end{array}\right.
$$

there is a unique solution $(\omega, \vartheta) \in \mathrm{E} \times \mathrm{E}$ with

$$
|(\mathrm{u}, \mathrm{y})(\mathrm{t})-(\omega, \vartheta)(\mathrm{t})| \leq C_{\Phi_{\mathrm{p}}, \Phi_{\mathrm{q}}} \Phi_{\mathrm{p}, \mathrm{q}}(\mathrm{t}) \epsilon
$$

Definition 2.6 ([35]) The proposed system (1.1) is generalized Ulam-Hyers-Rassias stable with respect to $\Phi_{\mathrm{p}, \mathrm{q}}=\left(\Phi_{\mathrm{p}}, \Phi_{\mathrm{q}}\right) \in \mathrm{C}(\mathrm{J}, \mathbb{R})$, if there is constant $C_{\Phi_{\mathrm{p}}, \Phi_{\mathrm{q}}}=\left(\mathrm{K}_{\Phi_{\mathrm{p}}}, \mathrm{K}_{\Phi_{\mathrm{q}}}\right)>0$, such that, for each $t \in J$ and solution $(u, y) \in E \times E$ of the following:

$$
\left\{\begin{array}{l}
\left|\mathrm{D}^{\mathrm{p}} \mathrm{u}(\mathrm{t})-\alpha\left(\mathrm{t}, \mathrm{y}(\mathrm{t}), \mathrm{D}^{\mathrm{p}} \mathrm{u}(\mathrm{t})\right)\right| \leq \Phi_{\mathrm{p}}(\mathrm{t}), \\
\left|\mathrm{D}^{\mathrm{q}} \mathrm{y}(\mathrm{t})-\chi\left(\mathrm{t}, \mathrm{u}(\mathrm{t}), \mathrm{D}^{\mathrm{q}} \mathrm{y}(\mathrm{t})\right)\right| \leq \Phi_{\mathrm{q}}(\mathrm{t}),
\end{array}\right.
$$

there is a unique solution $(\vartheta, \omega) \in \mathrm{E} \times \mathrm{E}$ of (1.1), which satisfies

$$
|(\mathrm{u}, \mathrm{y})(\mathrm{t})-(\omega, \vartheta)(\mathrm{t})| \leq C_{\Phi_{\mathrm{p}}, \Phi_{\mathrm{q}}} \Phi_{\mathrm{p}, \mathrm{q}}(\mathrm{t})
$$

Remark 2.1 We say that $(\mathrm{u}, \mathrm{y}) \in \mathrm{E} \times \mathrm{E}$ is a solution of $(2.1)$, if there are $\varphi_{\alpha}, \psi_{\chi} \in \mathrm{C}(\mathrm{J}, \mathbb{R})$, which depend upon $\mathrm{u}, \mathrm{y}$, respectively, such that

$\left(\mathrm{A}_{1}\right)\left|\varphi_{\alpha}(\mathrm{t})\right| \leq \epsilon_{\mathrm{p}},\left|\psi_{\chi}(\mathrm{t})\right| \leq \epsilon_{\mathrm{q}}, \mathrm{t} \in \mathrm{J}$; 
$\left(\mathrm{A}_{2}\right)$

$$
\begin{cases}D^{p} u(t)-\alpha\left(t, y(t), D^{p} u(t)\right)+\varphi_{\alpha}(t), & t \in J \\ D^{q} y(t)-\chi\left(t, u(t), D^{q} y(t)\right)+\psi_{\chi}(t), & t \in J\end{cases}
$$

\section{Existence and uniqueness}

In the current section, we set up conditions for the uniqueness and existence of solutions to the proposed system (1.1).

Lemma 3.1 Let $\varrho \in \mathrm{C}(\mathrm{J}, \mathbb{R})$, then, for $\mathrm{t} \in \mathrm{J}$, the equivalent Fredholm integral equation of the following boundary value problem:

$$
\left\{\begin{array}{l}
D^{p} u(t)=\varrho(t) ; \quad p \in(1,2] \\
\left.D^{p-2} u(t)\right|_{t=0^{+}}=\left.\gamma_{1} D^{p-2} u(t)\right|_{t=T^{-}} \\
\left.D^{p-1} u(t)\right|_{t=0^{+}}=\left.\beta_{1} D^{p-1} u(t)\right|_{t=T^{-}}
\end{array}\right.
$$

is given as

$$
u(t)=\int_{0}^{T} G_{p}(t, s) \varrho(s) d s
$$

where the Green's function $\mathrm{G}_{\mathrm{p}}(\mathrm{t}, \mathrm{s})$ is given as

$$
\mathrm{G}_{\mathrm{p}}(\mathrm{t}, \mathrm{s})= \begin{cases}\frac{1}{\Gamma(\mathrm{p})}(\mathrm{t}-\mathrm{s})^{\mathrm{p}-1}+\frac{\beta_{1} \mathrm{t}^{\mathrm{p}-1}}{\left(1-\beta_{1}\right) \Gamma(\mathrm{p})}+\frac{\gamma_{1} \mathrm{t}^{\mathrm{p}-2}\left[\mathrm{~T}-\left(1-\beta_{1}\right) \mathrm{s}\right]}{\left(1-\beta_{1}\right)\left(1-\gamma_{1}\right) \Gamma(\mathrm{p}-1)}, & 0 \leq \mathrm{s} \leq \mathrm{t} \leq \mathrm{T}, \\ \frac{\beta_{1} \mathrm{p}^{\mathrm{p}-1}}{\left(1-\beta_{1}\right) \Gamma(\mathrm{p})}+\frac{\gamma_{1} \mathrm{p}^{\mathrm{p}-2}\left[\mathrm{~T}-\left(1-\beta_{1}\right) s\right]}{\left(1-\beta_{1}\right)\left(1-\gamma_{1}\right) \Gamma(\mathrm{p}-1)}, & 0 \leq \mathrm{t} \leq \mathrm{s} \leq \mathrm{T}\end{cases}
$$

Proof For the proof, see Theorem 3.1 in [8].

So in view of Lemma 3.1, for $\mathrm{t} \in \mathrm{J}$, the solution of the proposed system (1.1) is equivalent to the toppled system of integral equations given by

$$
\left\{\begin{array}{l}
\mathrm{u}(\mathrm{t})=\int_{0}^{\mathrm{T}} \mathrm{G}_{\mathrm{p}}(\mathrm{t}, \mathrm{s}) \alpha\left(\mathrm{s}, \mathrm{y}(\mathrm{s}), \mathrm{D}^{\mathrm{p}} \mathrm{u}(\mathrm{s})\right) \mathrm{ds} \\
\mathrm{y}(\mathrm{t})=\int_{0}^{\mathrm{T}} \mathrm{G}_{\mathrm{q}}(\mathrm{t}, \mathrm{s}) \chi\left(\mathrm{s}, \mathrm{u}(\mathrm{s}), \mathrm{D}^{\mathrm{q}} \mathrm{y}(\mathrm{s})\right) \mathrm{ds} .
\end{array}\right.
$$

We use the following notation for convenience:

$$
\begin{aligned}
& \mathrm{v}(\mathrm{t})=\alpha\left(\mathrm{t}, \mathrm{y}(\mathrm{t}), \mathrm{D}^{\mathrm{p}} \mathrm{u}(\mathrm{t})\right)=\alpha(\mathrm{t}, \mathrm{y}(\mathrm{t}), \mathrm{v}(\mathrm{t})), \\
& \mathrm{z}(\mathrm{t})=\chi\left(\mathrm{t}, \mathrm{u}(\mathrm{t}), \mathrm{D}^{\mathrm{q}} \mathrm{y}(\mathrm{t})\right)=\chi(\mathrm{t}, \mathrm{u}(\mathrm{t}), \mathrm{z}(\mathrm{t}))
\end{aligned}
$$

Hence, for $t \in J$, (3.1) becomes

$$
\left\{\begin{array}{l}
u(t)=\int_{0}^{T} G_{p}(t, s) v(s) d s \\
y(t)=\int_{0}^{T} G_{q}(t, s) z(s) d s
\end{array}\right.
$$


where $v, z \in E$ satisfies the implicit functional equations and the Green's function $G_{q}(t, s)$ is given as

$$
\mathrm{G}_{\mathrm{q}}(\mathrm{t}, \mathrm{s})= \begin{cases}\frac{1}{\Gamma(\mathrm{q})}(\mathrm{t}-\mathrm{s})^{\mathrm{q}-1}+\frac{\beta_{2} \mathrm{t}^{\mathrm{q}-1}}{\left(1-\beta_{2}\right) \Gamma(\mathrm{q})}+\frac{\gamma_{2} \mathrm{t}^{\mathrm{q}-2}\left[\mathrm{~T}-\left(1-\beta_{2}\right) \mathrm{s}\right]}{\left(1-\beta_{2}\right)\left(1-\gamma_{2}\right) \Gamma(\mathrm{q}-1)}, & 0 \leq \mathrm{s} \leq \mathrm{t} \leq \mathrm{T}, \\ \frac{\beta_{2} \mathrm{q}^{\mathrm{q}-1}}{\left(1-\beta_{2}\right) \Gamma(\mathrm{q})}+\frac{\gamma_{2} \mathrm{q}^{-2}\left[\mathrm{~T}-\left(1-\beta_{2}\right) \mathrm{s}\right]}{\left(1-\beta_{2}\right)\left(1-\gamma_{2}\right) \Gamma(\mathrm{q}-1)}, & 0 \leq \mathrm{t} \leq \mathrm{s} \leq \mathrm{T} .\end{cases}
$$

Lemma 3.2 The Green's function $\mathrm{G}_{\mathrm{p}, \mathrm{q}}(\mathrm{t}, \mathrm{s})=\left(\mathrm{G}_{\mathrm{p}}(\mathrm{t}, \mathrm{s}), \mathrm{G}_{\mathrm{q}}(\mathrm{t}, \mathrm{s})\right)$ of the proposed system (1.1), has the properties given by:

(i) $G_{p, q}(t, s)$ is continuous over $\mathrm{J} \times \mathrm{J}$;

(ii) $\max _{\mathrm{t} \in J}\left|\mathrm{G}_{\mathrm{p}}(\mathrm{t}, \mathrm{s})\right| \leq \frac{1}{\Gamma(\mathrm{p})}(\mathrm{T}-\mathrm{s})^{\mathrm{p}-1}+\frac{\beta_{1} \mathrm{~T}^{\mathrm{p}-1}}{\left(1-\beta_{1}\right) \Gamma(\mathrm{p})}+\frac{\gamma_{1} \mathrm{~T}^{\mathrm{p}-2}\left[\mathrm{~T}-\left(1-\beta_{1}\right) \mathrm{s}\right]}{\left(1-\beta_{1}\right)\left(1-\gamma_{1}\right) \Gamma(\mathrm{p}-1)}=\mathrm{G}_{\mathrm{p}}(\mathrm{T}, \mathrm{s})$,

$\max _{\mathrm{t} \in \mathrm{J}}\left|\mathrm{G}_{\mathrm{q}}(\mathrm{t}, \mathrm{s})\right| \leq \frac{1}{\Gamma(\mathrm{q})}(\mathrm{T}-\mathrm{s})^{\mathrm{q}-1}+\frac{\beta_{2} \mathrm{~T}^{\mathrm{q}-1}}{\left(1-\beta_{2}\right) \Gamma(\mathrm{q})}+\frac{\gamma_{2} \mathrm{~T}^{\mathrm{q}-2}\left[\mathrm{~T}-\left(1-\beta_{2}\right) \mathrm{s}\right]}{\left(1-\beta_{2}\right)\left(1-\gamma_{2}\right) \Gamma(\mathrm{q}-1)}=\mathrm{G}_{\mathrm{q}}(\mathrm{T}, \mathrm{s}) ;$

(iii) $\max _{\mathrm{t} \in \mathrm{J}} \int_{0}^{\mathrm{T}}\left|\mathrm{G}_{\mathrm{p}}(\mathrm{t}, \mathrm{s})\right| d \mathrm{~s} \leq\left(\frac{\mathrm{T}^{\mathrm{p}}}{\Gamma(\mathrm{p}+1)}+\left|\frac{\beta_{1} \mathrm{~T}^{\mathrm{p}}}{\left(1-\beta_{1}\right) \Gamma(\mathrm{p})}\right|+\left|\frac{\gamma_{1}\left(1+\left|\beta_{1}\right|\right) \mathrm{T}^{\mathrm{p}}}{2\left(1-\beta_{1}\right)\left(1-\gamma_{1}\right) \Gamma(\mathrm{p}-1)}\right|\right), \mathrm{s} \in \mathrm{J}$,

$\max _{\mathrm{t} \in J} \int_{0}^{\mathrm{T}}\left|\mathrm{G}_{\mathrm{q}}(\mathrm{t}, \mathrm{s})\right| \mathrm{d} \mathrm{s} \leq\left(\frac{\mathrm{T}^{\mathrm{q}}}{\Gamma(\mathrm{q}+1)}+\left|\frac{\beta_{2} \mathrm{~T}^{\mathrm{q}}}{\left(1-\beta_{2}\right) \Gamma(\mathrm{q})}\right|+\left|\frac{\gamma_{2}\left(1+\left|\beta_{2}\right|\right) \mathrm{T}^{\mathrm{q}}}{2\left(1-\beta_{2}\right)\left(1-\gamma_{2}\right) \Gamma(\mathrm{q}-1)}\right|\right), \mathrm{s} \in \mathrm{J}$.

Proof It is very easy to prove (i), (ii) and (iii), the reader may refer to [8].

For computational convenience, we introduce the notations:

$$
\begin{aligned}
& \mathrm{N}_{\mathrm{p}}=\frac{\mathrm{T}^{\mathrm{p}}}{\Gamma(\mathrm{p}+1)}+\left|\frac{\beta_{1} \mathrm{~T}^{\mathrm{p}}}{\left(1-\beta_{1}\right) \Gamma(\mathrm{p})}\right|+\left|\frac{\gamma_{1}\left(1+\left|\beta_{1}\right|\right) \mathrm{T}^{\mathrm{p}}}{2\left(1-\beta_{1}\right)\left(1-\gamma_{1}\right) \Gamma(\mathrm{p}-1)}\right|, \\
& \mathrm{M}_{\mathrm{q}}=\frac{\mathrm{T}^{\mathrm{q}}}{\Gamma(\mathrm{q}+1)}+\left|\frac{\beta_{2} \mathrm{~T}^{\mathrm{q}}}{\left(1-\beta_{2}\right) \Gamma(\mathrm{q})}\right|+\left|\frac{\gamma_{2}\left(1+\left|\beta_{2}\right|\right) \mathrm{T}^{\mathrm{q}}}{2\left(1-\beta_{2}\right)\left(1-\gamma_{2}\right) \Gamma(\mathrm{q}-1)}\right|, \\
& \Omega_{\alpha}=\frac{\mathrm{K}_{\alpha}}{1-\mathrm{L}_{\alpha}}, \quad \Omega_{\chi}=\frac{\mathrm{K}_{\chi}}{1-\mathrm{L}_{\chi}} .
\end{aligned}
$$

If $\mathrm{u}, \mathrm{y}$ are the solutions of the proposed system (1.1) and $\mathrm{t} \in \mathrm{J}$, then

$$
\begin{aligned}
\mathrm{u}(\mathrm{t})= & \frac{1}{\Gamma(\mathrm{p})} \int_{0}^{\mathrm{t}}(\mathrm{t}-\mathrm{s})^{\mathrm{p}-1} \alpha(\mathrm{s}, \mathrm{y}(\mathrm{s}), \mathrm{v}(\mathrm{s})) \mathrm{d} \mathrm{s}+\frac{\beta_{1} \mathrm{t}^{\mathrm{p}-1}}{\left(1-\beta_{1}\right) \Gamma(\mathrm{p})} \int_{0}^{\mathrm{T}} \alpha(\mathrm{s}, \mathrm{y}(\mathrm{s}), \mathrm{v}(\mathrm{s})) \mathrm{d} \mathrm{s} \\
& +\frac{\gamma_{1} \mathrm{t}^{\mathrm{p}-2}}{\left(1-\beta_{1}\right)\left(1-\gamma_{1}\right) \Gamma(\mathrm{p}-1)} \int_{0}^{\mathrm{T}}\left[\mathrm{T}-\left(1-\beta_{1}\right) \mathrm{s}\right] \alpha(\mathrm{s}, \mathrm{u}(\mathrm{s}), \mathrm{v}(\mathrm{s})) \mathrm{d} \mathrm{s}
\end{aligned}
$$

and

$$
\begin{aligned}
\mathrm{y}(\mathrm{t})= & \frac{1}{\Gamma(\mathrm{q})} \int_{0}^{\mathrm{t}}(\mathrm{t}-\mathrm{s})^{\mathrm{q}-1} \chi(\mathrm{s}, \mathrm{u}(\mathrm{s}), \mathrm{z}(\mathrm{s})) \mathrm{d} \mathrm{s}+\frac{\beta_{2} \mathrm{t}^{\mathrm{q}-1}}{\left(1-\beta_{2}\right) \Gamma(\mathrm{q})} \int_{0}^{\mathrm{T}} \chi(\mathrm{s}, \mathrm{u}(\mathrm{s}), \mathrm{z}(\mathrm{s})) \mathrm{ds} \\
& +\frac{\gamma_{2} \mathrm{t}^{\mathrm{q}-2}}{\left(1-\beta_{2}\right)\left(1-\gamma_{2}\right) \Gamma(\mathrm{q}-1)} \int_{0}^{\mathrm{T}}\left[\mathrm{T}-\left(1-\beta_{2}\right) \mathrm{s}\right] \chi(\mathrm{s}, \mathrm{u}(\mathrm{s}), \mathrm{z}(\mathrm{s})) \mathrm{d} \mathrm{s} .
\end{aligned}
$$

Now, we transform the proposed system (1.1) into a fixed point problem. Let an operator $\mathrm{T}: \mathrm{E} \times \mathrm{E} \rightarrow \mathrm{E} \times \mathrm{E}$ be defined as

$$
T(u, y)(t)=\left(\begin{array}{c}
\int_{0}^{T} G_{p}(t, s) \alpha(t, y(s), v(s)) d s \\
\int_{0}^{T} G_{q}(t, s) \chi(t, u(s), z(s)) d s
\end{array}\right)=\left(\begin{array}{c}
T_{p}(y, v)(t) \\
T_{q}(u, z)(t)
\end{array}\right) .
$$


Then the solution of (1.1) coincides with the fixed point of T, where

$$
\begin{aligned}
\mathrm{T}_{\mathrm{p}}(\mathrm{u})(\mathrm{t})= & \frac{1}{\Gamma(\mathrm{p})} \int_{0}^{\mathrm{t}}(\mathrm{t}-\mathrm{s})^{\mathrm{p}-1} \mathrm{v}(\mathrm{s}) \mathrm{d} \mathrm{s}+\frac{\beta_{1} \mathrm{t}^{\mathrm{p}-1}}{\left(1-\beta_{1}\right) \Gamma(\mathrm{p})} \int_{0}^{\mathrm{T}} \mathrm{v}(\mathrm{s}) \mathrm{ds} \\
& +\frac{\gamma_{1} \mathrm{t}^{\mathrm{p}-2}}{\left(1-\beta_{1}\right)\left(1-\gamma_{1}\right) \Gamma(\mathrm{p}-1)} \int_{0}^{\mathrm{T}}\left[\mathrm{T}-\left(1-\beta_{1}\right) \mathrm{s}\right] \mathrm{v}(\mathrm{s}) \mathrm{ds}
\end{aligned}
$$

and

$$
\begin{aligned}
\mathrm{T}_{\mathrm{q}}(\mathrm{y})(\mathrm{t})= & \frac{1}{\Gamma(\mathrm{q})} \int_{0}^{\mathrm{t}}(\mathrm{t}-\mathrm{s})^{\mathrm{q}-1} \mathrm{z}(\mathrm{s}) \mathrm{d} \mathrm{s}+\frac{\beta_{1} \mathrm{t}^{\mathrm{q}-1}}{\left(1-\beta_{2}\right) \Gamma(\mathrm{q})} \int_{0}^{\mathrm{T}} \mathrm{z}(\mathrm{s}) \mathrm{ds} \\
& +\frac{\gamma_{2} \mathrm{t}^{\mathrm{q}-2}}{\left(1-\beta_{2}\right)\left(1-\gamma_{2}\right) \Gamma(\mathrm{q}-1)} \int_{0}^{\mathrm{T}}\left[\mathrm{T}-\left(1-\beta_{2}\right) \mathrm{s}\right] \mathrm{z}(\mathrm{s}) \mathrm{ds} .
\end{aligned}
$$

For further analysis, the following hypotheses need to hold:

$\left(\mathrm{H}_{1}\right)$ For $\mathrm{t} \in \mathrm{J}$ and $\mathrm{y}, \mathrm{v} \in \mathbb{R}$, there are $\mathrm{a}_{1}, \mathrm{~b}_{1}, \mathrm{c}_{1} \in \mathrm{C}\left(\mathrm{J}, \mathbb{R}^{+}\right)$, such that

$$
|\alpha(\mathrm{t}, \mathrm{y}(\mathrm{t}), \mathrm{v}(\mathrm{t}))| \leq \mathrm{a}_{1}(\mathrm{t})+\mathrm{b}_{1}(\mathrm{t})|\mathrm{y}(\mathrm{t})|+\mathrm{c}_{1}(\mathrm{t})|\mathrm{v}(\mathrm{t})|
$$

with $a_{1}^{*}=\sup _{t \in J} a_{1}(t), b_{1}^{*}=\sup _{t \in J} b_{1}(t)$ and $c_{1}^{*}=\sup _{t \in J} c_{1}(t)<1$.

Similarly, for $t \in J$ and $u, z \in \mathbb{R}$, there are $a_{1}, b_{2}, c_{2} \in C\left(J, \mathbb{R}^{+}\right)$, such that

$$
|\chi(\mathrm{t}, \mathrm{u}(\mathrm{t}), \mathrm{z}(\mathrm{t}))| \leq \mathrm{a}_{2}(\mathrm{t})+\mathrm{b}_{2}(\mathrm{t})|\mathrm{u}(\mathrm{t})|+\mathrm{c}_{2}(\mathrm{t})|\mathrm{z}(\mathrm{t})|
$$

with $a_{2}^{*}=\sup _{t \in J} a_{2}(t), b_{2}^{*}=\sup _{t \in J} b_{2}(t)$ and $c_{2}^{*}=\sup _{t \in J} c_{2}(t)<1$.

$\left(\mathrm{H}_{2}\right)$ For all $\mathrm{y}, \mathrm{v}, \overline{\mathrm{y}}, \overline{\mathrm{v}} \in \mathbb{R}$ and for each $\mathrm{t} \in \mathrm{J}$ there exist constants $\mathrm{K}_{\alpha}>0,0<\mathrm{L}_{\alpha}<1$, such that

$$
|\alpha(\mathrm{t}, \mathrm{y}, \mathrm{v})-\alpha(\mathrm{t}, \overline{\mathrm{y}}, \overline{\mathrm{v}})| \leq \mathrm{K}_{\alpha}|\mathrm{y}-\overline{\mathrm{y}}|+\mathrm{L}_{\alpha}|\mathrm{v}-\overline{\mathrm{v}}|
$$

Similarly, for all $\mathrm{u}, \mathrm{z}, \overline{\mathrm{u}}, \overline{\mathrm{z}} \in \mathbb{R}$ and for each $\mathrm{t} \in \mathrm{J}$ there exist constants $\mathrm{K}_{\chi}>0,0<\mathrm{L}_{\chi}<$ 1 , such that

$$
|\chi(\mathrm{t}, \mathrm{u}, \mathrm{z})-\chi(\mathrm{t}, \overline{\mathrm{u}}, \overline{\mathrm{z}})| \leq \mathrm{K}_{\chi}|\mathrm{u}-\overline{\mathrm{u}}|+\mathrm{L}_{\chi}|\mathrm{z}-\overline{\mathrm{z}}|
$$

Theorem 3.1 Let $\alpha, \chi: J \times \mathbb{R} \times \mathbb{R} \rightarrow \mathbb{R}$ and $\left(H_{1}\right)$ hold. Then the operator $\mathrm{T}: \check{\mathbb{C}} \rightarrow \check{C}$ defined in (3.2) is completely continuous.

Proof In view of continuity of $\alpha, \chi$ and $G_{p, q}(t, s), T$ is also continuous for all $(\mathrm{y}, \mathrm{z}) \in \check{\mathbb{C}}$. Suppose $\mathscr{B} \subseteq \check{C}$ is a bounded set. So, for every $\mathrm{y} \in \mathscr{B}$, we have

$$
\begin{aligned}
\left|\mathrm{T}_{\mathrm{p}}(\mathrm{u})(\mathrm{t})\right|= & \mid \frac{1}{\Gamma(\mathrm{p})} \int_{0}^{\mathrm{t}}(\mathrm{t}-\mathrm{s})^{\mathrm{p}-1} \mathrm{v}(\mathrm{s}) \mathrm{ds}+\frac{\beta_{1} \mathrm{t}^{\mathrm{p}-1}}{\left(1-\beta_{1}\right) \Gamma(\mathrm{p})} \int_{0}^{\mathrm{T}} \mathrm{v}(\mathrm{s}) \mathrm{ds} \\
& +\frac{\gamma_{1} \mathrm{t}^{\mathrm{p}-2}}{\left(1-\beta_{1}\right)\left(1-\gamma_{1}\right) \Gamma(\mathrm{p}-1)} \int_{0}^{\mathrm{T}}\left[\mathrm{T}-\left(1-\beta_{1}\right) \mathrm{s}\right] \mathrm{v}(\mathrm{s}) \mathrm{ds} \mid
\end{aligned}
$$


Now by $\left(\mathrm{H}_{1}\right)$ with $\|\mathrm{y}\| \leq \xi_{\mathrm{p}}$, then

$$
\begin{aligned}
|\mathrm{v}(\mathrm{t})| & =|\alpha(\mathrm{t}, \mathrm{y}(\mathrm{t}), \mathrm{v}(\mathrm{t}))| \\
& \leq \mathrm{a}_{1}(\mathrm{t})+\mathrm{b}_{1}(\mathrm{t})|\mathrm{y}(\mathrm{t})|+\mathrm{c}_{1}(\mathrm{t})|\mathrm{v}(\mathrm{t})| \\
& \leq \mathrm{a}_{1}^{*}+\mathrm{b}_{1}^{*} \xi_{\mathrm{p}}+\mathrm{c}_{1}^{*}\|\mathrm{v}\|_{\mathrm{E}} .
\end{aligned}
$$

So, we obtain

$$
\|\mathrm{v}\|_{\mathrm{E}} \leq \frac{\mathrm{a}_{1}^{*}+\mathrm{b}_{1}^{*} \xi_{\mathrm{p}}}{1-\mathrm{c}_{1}^{*}}=\Upsilon_{\mathrm{p}}
$$

Now by using (iii) of Lemma 3.2 and (3.4) in (3.3), we get

$$
\left\|T_{p}(u)\right\|_{E} \leq N_{p} \cdot \Upsilon_{p}
$$

In the same fashion, we obtain

$$
\left\|\mathrm{T}_{\mathrm{q}}(\mathrm{y})\right\|_{\mathrm{E}} \leq \mathrm{M}_{\mathrm{q}} \cdot \Upsilon_{\mathrm{q}}
$$

where

$$
\Upsilon_{\mathrm{q}}=\frac{\mathrm{a}_{2}^{*}+\mathrm{b}_{2}^{*} \xi_{\mathrm{q}}}{1-\mathrm{c}_{2}^{*}}
$$

with $\|\mathrm{u}\| \leq \xi_{\mathrm{q}}$. Thus from (3.5) and (3.6), we get

$$
\left\|T_{p}(u)\right\|_{E}+\left\|T_{q}(y)\right\|_{E} \leq N_{p} \cdot \Upsilon_{p}+M_{q} \cdot \Upsilon_{q}=\mathscr{M}_{0},
$$

which yields

$$
\|\mathrm{T}(\mathrm{u}, \mathrm{y})\|_{\mathrm{E} \times \mathrm{E}} \leq \mathscr{M}_{0}
$$

Thus, $\mathrm{T}$ is uniformly bounded. Now we prove the operator $\mathrm{T}$ is equi-continuous. For this purpose, suppose $\mathrm{t}_{1}<\mathrm{t}_{2} \in \mathrm{J}$ and $\mathrm{u} \in \mathscr{B}$, then

$$
\begin{aligned}
& \left|\mathrm{T}_{\mathrm{p}}(\mathrm{u})\left(\mathrm{t}_{1}\right)-\mathrm{T}_{\mathrm{p}}(\mathrm{u})\left(\mathrm{t}_{2}\right)\right| \\
& =\mid \frac{1}{\Gamma(\mathrm{p})} \int_{0}^{\mathrm{t}_{1}}\left[\left(\mathrm{t}_{1}-\mathrm{s}\right)^{\mathrm{p}-1}-\left(\mathrm{t}_{2}-\mathrm{s}\right)^{\mathrm{p}-1}\right] \mathrm{v}(\mathrm{s}) \mathrm{ds}-\frac{1}{\Gamma(\mathrm{p})} \int_{\mathrm{t}_{1}}^{\mathrm{t}_{2}}\left(\mathrm{t}_{2}-\mathrm{s}\right)^{\mathrm{p}-1} \mathrm{v}(\mathrm{s}) \mathrm{ds} \\
& \quad+\frac{\beta_{1}\left(\mathrm{t}_{1}^{\mathrm{p}-1}-\mathrm{t}_{2}^{\mathrm{p}-1}\right)}{\left(1-\beta_{1}\right) \Gamma(\mathrm{p}-1)} \int_{0}^{\mathrm{T}} \mathrm{v}(\mathrm{s}) \mathrm{ds} \mid \\
& \leq \Upsilon_{\mathrm{p}}\left(\left|\frac{1}{\Gamma(\mathrm{p}+1)}\left[2\left(\mathrm{t}_{2}-\mathrm{t}_{1}\right)^{\mathrm{p}}-\left(\mathrm{t}_{2}^{\mathrm{p}}-\mathrm{t}_{1}^{\mathrm{p}}\right)\right]\right|+\left|\frac{\beta_{1}\left(\mathrm{t}_{2}^{\mathrm{p}-1}-\mathrm{t}_{1}^{\mathrm{p}-1}\right) \mathrm{T}}{\left(1-\beta_{1}\right) \Gamma(\mathrm{p}-1)}\right|\right) .
\end{aligned}
$$

In the same fashion, we can show that

$$
\begin{aligned}
& \left|\mathrm{T}_{\mathrm{q}}(\mathrm{y})\left(\mathrm{t}_{1}\right)-\mathrm{T}_{\mathrm{q}}(\mathrm{y})\left(\mathrm{t}_{2}\right)\right| \\
& \quad \leq \Upsilon_{\mathrm{q}}\left(\left|\frac{1}{\Gamma(\mathrm{q}+1)}\left[2\left(\mathrm{t}_{2}-\mathrm{t}_{1}\right)^{\mathrm{q}}-\left(\mathrm{t}_{2}^{\mathrm{q}}-\mathrm{t}_{1}^{\mathrm{q}}\right)\right]\right|+\left|\frac{\beta_{2}\left(\mathrm{t}_{2}^{\mathrm{q}-1}-\mathrm{t}_{1}^{\mathrm{q}-1}\right) \mathrm{T}}{\left(1-\beta_{2}\right) \Gamma(\mathrm{q}-1)}\right|\right) .
\end{aligned}
$$


The right hand sides of (3.7) and (3.8) approach zero, when $t_{1} \rightarrow t_{2}$. So by the ArzelaAscoli theorem, we infer that $\mathrm{T}$ is equi-continuous and uniformly equi-continuous. Also, it is very easy to prove $\mathrm{T}(\mathscr{B}) \subset \mathscr{B}$. Therefore, $\mathrm{T}$ is completely continuous.

Theorem 3.2 Under the hypothesis $\left(\mathrm{H}_{2}\right)$ and

$$
\mathrm{N}_{\mathrm{p}} \cdot \Omega_{\alpha}+\mathrm{M}_{\mathrm{q}} \cdot \Omega_{\chi}<1
$$

The proposed system (1.1) has a unique solution.

Proof Let $\mathrm{u}, \overline{\mathrm{u}} \in \check{\mathbb{C}}$ and consider

$$
\begin{aligned}
& \left|\mathrm{T}_{\mathrm{p}}(\mathrm{u})(\mathrm{t})-\mathrm{T}_{\mathrm{p}}(\overline{\mathrm{u}})(\mathrm{t})\right| \\
& =\mid \frac{1}{\Gamma(\mathrm{p})} \int_{0}^{\mathrm{t}}(\mathrm{t}-\mathrm{s})^{\mathrm{p}-1} \mathrm{ds}+\frac{\beta_{1} \mathrm{t}^{\mathrm{p}-1}}{\left(1-\beta_{1}\right) \Gamma(\mathrm{p})} \int_{0}^{\mathrm{T}} \mathrm{ds} \\
& \quad+\frac{\gamma_{1} \mathrm{t}^{\mathrm{p}-2}}{\left(1-\beta_{1}\right)\left(1-\gamma_{1}\right) \Gamma(\mathrm{p}-1)} \int_{0}^{\mathrm{T}}\left[\mathrm{T}-\left(1-\beta_{1}\right) \mathrm{s}\right] \mathrm{ds}|| \mathrm{v}(\mathrm{s})-\overline{\mathrm{v}}(\mathrm{s}) \mid
\end{aligned}
$$

where

$$
\begin{aligned}
& \mathrm{v}(\mathrm{t})=\alpha(\mathrm{t}, \mathrm{y}(\mathrm{t}), \mathrm{v}(\mathrm{t})) \\
& \overline{\mathrm{v}}(\mathrm{t})=\alpha(\mathrm{t}, \overline{\mathrm{y}}(\mathrm{t}), \overline{\mathrm{v}}(\mathrm{t}))
\end{aligned}
$$

By using $\left(\mathrm{H}_{2}\right)$

$$
\begin{aligned}
|\mathrm{v}(\mathrm{t})-\overline{\mathrm{v}}(\mathrm{t})| & =|\alpha(\mathrm{t}, \mathrm{y}(\mathrm{t}), \mathrm{v}(\mathrm{t}))-\alpha(\mathrm{t}, \overline{\mathrm{y}}(\mathrm{t}), \overline{\mathrm{v}}(\mathrm{t}))| \\
& \leq \mathrm{K}_{\alpha}|\mathrm{y}(\mathrm{t})-\overline{\mathrm{y}}(\mathrm{t})|+\mathrm{L}_{\alpha}|\mathrm{v}(\mathrm{t})-\overline{\mathrm{v}}(\mathrm{t})|,
\end{aligned}
$$

we obtain

$$
|\mathrm{v}(\mathrm{t})-\overline{\mathrm{v}}(\mathrm{t})| \leq \Omega_{\alpha}|\mathrm{y}(\mathrm{t})-\overline{\mathrm{y}}(\mathrm{t})|
$$

Put (3.11) in (3.10) and taking a maximum over J, we get

$$
\left\|\mathrm{T}_{\mathrm{p}}(\mathrm{u})-\mathrm{T}_{\mathrm{p}}(\overline{\mathrm{u}})\right\|_{\mathrm{E}} \leq\left(\mathrm{N}_{\mathrm{p}} \cdot \Omega_{\alpha}\right)\|\mathrm{y}-\overline{\mathrm{y}}\|_{\mathrm{E}} .
$$

In the same fashion, we can obtain

$$
\left\|\mathrm{T}_{\mathrm{q}}(\mathrm{y})-\mathrm{T}_{\mathrm{q}}(\overline{\mathrm{y}})\right\|_{\mathrm{E}} \leq\left(\mathrm{M}_{\mathrm{q}} \cdot \Omega_{\chi}\right)\|\mathrm{u}-\overline{\mathrm{u}}\|_{\mathrm{E}}
$$

So from (3.12) and (3.13), we get

$$
\|\mathrm{T}(\mathrm{u}, \mathrm{y})-\mathrm{T}(\overline{\mathrm{u}}, \overline{\mathrm{y}})\|_{\mathrm{E} \times \mathrm{E}} \leq\left(\mathrm{N}_{\mathrm{p}} \cdot \Omega_{\alpha}+\mathrm{M}_{\mathrm{q}} \cdot \Omega_{\chi}\right)\|(\mathrm{u}, \mathrm{y})-(\overline{\mathrm{u}}, \overline{\mathrm{y}})\|_{\mathrm{E} \times \mathrm{E}} \cdot
$$

Thus, T is contraction. Therefore, by Banach's contraction principle, $\mathrm{T}$ has a fixed point. So, we infer that the proposed toppled system (1.1) has a unique solution. 
Theorem 3.3 In view of continuity of the functions $\alpha, \chi$ and supposing $\left(\mathrm{H}_{1}\right)$ :

$$
\begin{aligned}
\left(\mathrm{H}_{3}\right) \mathbb{A}_{1} & =\int_{0}^{\mathrm{T}} \mathrm{G}_{\mathrm{p}}(\mathrm{T}, \mathrm{s}) \mathrm{a}_{1}(\mathrm{~s}) \mathrm{ds}, \mathbb{B}_{1}=\int_{0}^{\mathrm{T}} \mathrm{G}_{\mathrm{p}}(\mathrm{T}, \mathrm{s})\left[\mathrm{b}_{1}(\mathrm{~s})+\mathrm{c}_{1}(\mathrm{~s})\right] \mathrm{d} \mathrm{s}<1, \\
\mathbb{A}_{2} & =\int_{0}^{\mathrm{T}} \mathrm{G}_{\mathrm{q}}(\mathrm{T}, \mathrm{s}) \mathrm{a}_{2}(\mathrm{~s}) \mathrm{d} \mathrm{s}, \mathbb{B}_{2}=\int_{0}^{\mathrm{T}} \mathrm{G}_{\mathrm{q}}(\mathrm{T}, \mathrm{s})\left[\mathrm{b}_{2}(\mathrm{~s})+\mathrm{c}_{2}(\mathrm{~s})\right] \mathrm{d} \mathrm{s}<1
\end{aligned}
$$

hold. Then the proposed system (1.1) has at least one solution.

Proof Let a set $\mathfrak{D}$, define as

$$
\mathfrak{D}=\left\{(\mathrm{u}, \mathrm{y}) \in \mathrm{E} \times \mathrm{E}:\|(\mathrm{u}, \mathrm{y})\|_{\mathrm{E} \times \mathrm{E}}<R_{\mathfrak{D}}\right\}
$$

where $\max \left\{\frac{2 \mathbb{A}_{1}}{1-2 \mathbb{B}_{1}}, \frac{2 \mathbb{A}_{2}}{1-2 \mathbb{B}_{2}}\right\}<R_{\mathfrak{D}}$. Furthermore, the operator defined by $\mathrm{T}: \overline{\mathfrak{D}} \rightarrow \check{C}$ in $(3.2)$ is completely continuous. Suppose $(\mathrm{u}, \mathrm{y}) \in \mathfrak{D}$ then, by definition of $\mathfrak{D}$, we have $\|(\mathrm{u}, \mathrm{y})\|_{\mathrm{E} \times \mathrm{E}}<$ $R_{\mathfrak{D}}$

$$
\begin{aligned}
\left\|T_{p}(y, v)\right\|_{E} \leq & \max _{t \in J} \int_{0}^{T}\left|G_{p}(t, s)\right||\alpha(s, y(s), v(s))| d s \\
\leq & \max _{t \in J} \int_{0}^{T}\left|G_{p}(t, s)\right| a_{1}(s) d s \\
& +\max _{t \in J} \int_{0}^{T}\left|G_{p}(t, s)\right|\left[b_{1}(s)|y(s)|+c_{1}(s)|v(s)|\right] d s \\
\leq & \int_{0}^{T} G_{p}(T, s) a_{1}(s) d s+R_{\mathfrak{D}} \int_{0}^{T} G_{p}(T, s)\left[b_{1}(s)+c_{1}(s)\right] d s \\
= & \mathbb{A}_{1}+R_{\mathfrak{D}} \mathbb{B}_{1} \leq \frac{R_{\mathfrak{D}}}{2} .
\end{aligned}
$$

Also

$$
\left\|\mathrm{T}_{\mathrm{q}}(\mathrm{y}, \mathrm{v})\right\|_{\mathrm{E}} \leq \frac{R_{\mathfrak{D}}}{2}
$$

Therefore,

$$
\|\mathrm{T}(\mathrm{u}, \mathrm{y})\|_{\mathrm{E} \times \mathrm{E}} \leq R_{\mathfrak{D}} .
$$

So $T(y, z) \in \overline{\mathfrak{D}}$. Thus, in the light of Theorem 3.1, $\mathrm{T}: \overline{\mathfrak{D}} \rightarrow \overline{\mathfrak{D}}$ is completely continuous. Now, we consider an eigenvalue problem defined as

$$
(\mathrm{u}, \mathrm{y})=\delta \mathrm{T}(\mathrm{u}, \mathrm{y}), \quad 0<\delta<1
$$

So in view of the solution $(u, y)$ of (3.14), we obtain

$$
\begin{aligned}
\|\mathrm{u}\|_{\mathrm{E}} & =\left\|\delta \mathrm{T}_{\mathrm{p}}(\mathrm{y}, \mathrm{v})\right\|_{\mathrm{E}} \\
& \leq \max _{\mathrm{t} \in J} \int_{0}^{\mathrm{T}}\left|\mathrm{G}_{\mathrm{p}}(\mathrm{t}, \mathrm{s})\right||\alpha(\mathrm{s}, \mathrm{y}(\mathrm{s}), \mathrm{v}(\mathrm{s}))| \mathrm{d} s \\
& \leq \max _{\mathrm{t} \in J} \int_{0}^{\mathrm{T}}\left|\mathrm{G}_{\mathrm{p}}(\mathrm{t}, \mathrm{s})\right| \mathrm{a}_{1}(\mathrm{~s}) \mathrm{d} \mathrm{s}+\max _{\mathrm{t} \in J} \int_{0}^{\mathrm{T}}\left|\mathrm{G}_{\mathrm{p}}(\mathrm{t}, \mathrm{s})\right|\left[\mathrm{b}_{1}(\mathrm{~s})|\mathrm{y}(\mathrm{s})|+\mathrm{c}_{1}(\mathrm{~s})|\mathrm{v}(\mathrm{s})|\right] \mathrm{d} \mathrm{s}
\end{aligned}
$$




$$
\begin{aligned}
& \leq \int_{0}^{\mathrm{T}} \mathrm{G}_{\mathrm{p}}(\mathrm{T}, \mathrm{s}) \mathrm{a}_{1}(\mathrm{~s}) \mathrm{d} \mathrm{s}+R_{\mathcal{D}} \int_{0}^{\mathrm{T}} \mathrm{G}_{\mathrm{p}}(\mathrm{T}, \mathrm{s})\left[\mathrm{b}_{1}(\mathrm{~s})+\mathrm{c}_{1}(\mathrm{~s})\right] \mathrm{d} \mathrm{s} \\
& =\mathbb{A}_{1}+R_{\mathfrak{D}} \mathbb{B}_{1} \leq \frac{R_{\mathfrak{D}}}{2} .
\end{aligned}
$$

Similarly

$$
\|\mathrm{y}\|_{\mathrm{E}} \leq \frac{R_{\mathfrak{D}}}{2} .
$$

Thus

$$
\|(\mathrm{u}, \mathrm{y})\|_{\mathrm{E} \times \mathrm{E}} \leq R_{\mathfrak{D}} .
$$

From equation (3.15), we get $(\mathrm{u}, \mathrm{y}) \notin \partial \mathfrak{D}$. So, in view of Theorem 2.1 , T has at least one fixed point lies in $\overline{\mathfrak{D}}$. This shows there is at least one solution of the proposed system (1.1).

\section{Stability results}

In this section, we will investigate the stability results in the sense of Ulam for the proposed system (1.1).

Lemma 4.1 Consider $(\mathrm{u}, \mathrm{y}) \in \mathrm{E} \times \mathrm{E}$ be the solution of (2.1), then for $\mathrm{t} \in \mathrm{J}$ we have

$$
\left\{\begin{array}{l}
|\mathrm{u}(\mathrm{t})-\mathrm{m}(\mathrm{t})| \leq \mathrm{N}_{\mathrm{p}} \epsilon_{\mathrm{p}} \\
|\mathrm{y}(\mathrm{t})-\mathrm{n}(\mathrm{t})| \leq \mathrm{M}_{\mathrm{q}} \epsilon_{\mathrm{q}}
\end{array}\right.
$$

Proof By $\left(\mathrm{A}_{2}\right)$ of Remark 2.1 and for $\mathrm{t} \in \mathrm{J}$, we have

$$
\left\{\begin{array}{l}
\mathrm{D}^{p} \mathrm{u}(\mathrm{t})=\alpha\left(\mathrm{t}, \mathrm{y}(\mathrm{t}), \mathrm{D}^{\mathrm{p}} \mathrm{u}(\mathrm{t})\right)+\varphi_{\alpha}(\mathrm{t}), \\
\mathrm{D}^{q} \mathrm{y}(\mathrm{t})=\chi\left(\mathrm{t}, \mathrm{u}(\mathrm{t}), \mathrm{D}^{\mathrm{q}} \mathrm{y}(\mathrm{t})\right)+\psi_{\chi}(\mathrm{t}), \\
\left.\mathrm{D}^{\mathrm{p}-2} \mathrm{u}(\mathrm{t})\right|_{\mathrm{t}=0^{+}}=\left.\gamma_{1} \mathrm{D}^{\mathrm{p}-2} \mathrm{u}(\mathrm{t})\right|_{\mathrm{t}=\mathrm{T}^{-}},\left.\quad \mathrm{D}^{\mathrm{p}-1} \mathrm{u}(\mathrm{t})\right|_{\mathrm{t}=0^{+}}=\left.\beta_{1} \mathrm{D}^{\mathrm{p}-1} \mathrm{u}(\mathrm{t})\right|_{\mathrm{t}=\mathrm{T}^{-}}, \\
\left.\mathrm{D}^{\mathrm{q}-2} \mathrm{y}(\mathrm{t})\right|_{\mathrm{t}=0^{+}}=\left.\gamma_{2} \mathrm{D}^{\mathrm{q}-2} \mathrm{y}(\mathrm{t})\right|_{\mathrm{t}=\mathrm{T}^{-}},\left.\quad \mathrm{D}^{\mathrm{q}-1} \mathrm{y}(\mathrm{t})\right|_{\mathrm{t}=0^{+}}=\left.\beta_{2} \mathrm{D}^{\mathrm{q}-1} \mathrm{y}(\mathrm{t})\right|_{\mathrm{t}=\mathrm{T}^{-}}
\end{array}\right.
$$

So in view of Lemma 2.1, for $t \in J$ the solution of (4.1) will be of the given form,

$$
\left\{\begin{array}{l}
\mathrm{u}(\mathrm{t})=\int_{0}^{\mathrm{T}} \mathrm{G}_{\mathrm{p}}(\mathrm{t}, \mathrm{s}) \alpha\left(\mathrm{s}, \mathrm{y}(\mathrm{s}), \mathrm{D}^{\mathrm{p}} \mathrm{u}(\mathrm{s})\right) \mathrm{d} \mathrm{s}+\int_{0}^{\mathrm{T}} \mathrm{G}_{\mathrm{p}}(\mathrm{t}, \mathrm{s}) \varphi_{\alpha}(\mathrm{s}) \mathrm{ds}, \\
\mathrm{y}(\mathrm{t})=\int_{0}^{\mathrm{T}} \mathrm{G}_{\mathrm{q}}(\mathrm{t}, \mathrm{s}) \chi\left(\mathrm{s}, \mathrm{u}(\mathrm{s}), \mathrm{D}^{\mathrm{q}} \mathrm{y}(\mathrm{s})\right) \mathrm{d} \mathrm{s}+\int_{0}^{\mathrm{T}} \mathrm{G}_{\mathrm{q}}(\mathrm{t}, \mathrm{s}) \psi_{\chi}(\mathrm{s}) \mathrm{d} \mathrm{s} .
\end{array}\right.
$$

From the first equation of system (4.2), we have

$$
\begin{aligned}
\mathrm{u}(\mathrm{t})= & \frac{1}{\Gamma(\mathrm{p})} \int_{0}^{\mathrm{t}}(\mathrm{t}-\mathrm{s})^{\mathrm{p}-1} \mathrm{v}(\mathrm{s}) \mathrm{d} \mathrm{s}+\frac{\beta_{1} \mathrm{t}^{\mathrm{p}-1}}{\left(1-\beta_{1}\right) \Gamma(\mathrm{p})} \int_{0}^{\mathrm{T}} \mathrm{v}(\mathrm{s}) \mathrm{d} \mathrm{s} \\
& +\frac{\gamma_{1} \mathrm{t}^{\mathrm{p}-2}}{\left(1-\beta_{1}\right)\left(1-\gamma_{1}\right) \Gamma(\mathrm{p}-1)} \int_{0}^{\mathrm{T}}\left[\mathrm{T}-\left(1-\beta_{1}\right) \mathrm{s}\right] \mathrm{v}(\mathrm{s}) \mathrm{d} \mathrm{s} \\
& +\frac{1}{\Gamma(\mathrm{p})} \int_{0}^{\mathrm{t}}(\mathrm{t}-\mathrm{s})^{\mathrm{p}-1} \varphi_{\alpha}(\mathrm{s}) \mathrm{d} \mathrm{s}+\frac{\beta_{1} \mathrm{t}^{\mathrm{p}-1}}{\left(1-\beta_{1}\right) \Gamma(\mathrm{p})} \int_{0}^{\mathrm{T}} \varphi_{\alpha}(\mathrm{s}) \mathrm{d} \mathrm{s} \\
& +\frac{\gamma_{1} \mathrm{t}^{\mathrm{p}-2}}{\left(1-\beta_{1}\right)\left(1-\gamma_{1}\right) \Gamma(\mathrm{p}-1)} \int_{0}^{\mathrm{T}}\left[\mathrm{T}-\left(1-\beta_{1}\right) \mathrm{s}\right] \varphi_{\alpha}(\mathrm{s}) \mathrm{ds} .
\end{aligned}
$$


For computational convenience, we use $\mathrm{m}(\mathrm{t})$ for the sum of terms which are free of $\varphi_{\alpha}$, so we have

$$
\begin{aligned}
\mathrm{m}(\mathrm{t})= & \frac{1}{\Gamma(\mathrm{p})} \int_{0}^{\mathrm{t}}(\mathrm{t}-\mathrm{s})^{\mathrm{p}-1} \mathrm{v}(\mathrm{s}) \mathrm{ds}+\frac{\beta_{1} \mathrm{t}^{\mathrm{p}-1}}{\left(1-\beta_{1}\right) \Gamma(\mathrm{p})} \int_{0}^{\mathrm{T}} \mathrm{v}(\mathrm{s}) \mathrm{ds} \\
& +\frac{\gamma_{1} \mathrm{t}^{\mathrm{p}-2}}{\left(1-\beta_{1}\right)\left(1-\gamma_{1}\right) \Gamma(\mathrm{p}-1)} \int_{0}^{\mathrm{T}}\left[\mathrm{T}-\left(1-\beta_{1}\right) \mathrm{s}\right] \mathrm{v}(\mathrm{s}) \mathrm{ds} .
\end{aligned}
$$

So from the above and taking the absolute value, (4.3) becomes

$$
\begin{aligned}
|\mathrm{z}(\mathrm{t})-\mathrm{m}(\mathrm{t})| \leq & \mid \frac{1}{\Gamma(\mathrm{p})} \int_{0}^{\mathrm{t}}(\mathrm{t}-\mathrm{s})^{\mathrm{p}-1} \varphi_{\alpha}(\mathrm{s}) \mathrm{d} \mathrm{s}+\frac{\beta_{1} \mathrm{t}^{\mathrm{p}-1}}{\left(1-\beta_{1}\right) \Gamma(\mathrm{p})} \int_{0}^{\mathrm{T}} \varphi_{\alpha}(\mathrm{s}) \mathrm{d} \mathrm{s} \\
& +\frac{\gamma_{1} \mathrm{t}^{\mathrm{p}-2}}{\left(1-\beta_{1}\right)\left(1-\gamma_{1}\right) \Gamma(\mathrm{p}-1)} \int_{0}^{\mathrm{T}}\left[\mathrm{T}-\left(1-\beta_{1}\right) \mathrm{s}\right] \varphi_{\alpha}(\mathrm{s}) \mathrm{ds} \mid
\end{aligned}
$$

Using (iii) of Lemma 3.2 and $\left(\mathrm{A}_{1}\right)$ of Lemma 2.1, we get

$$
|\mathrm{u}(\mathrm{t})-\mathrm{m}(\mathrm{t})| \leq \mathrm{N}_{\mathrm{p}} \epsilon_{\mathrm{p}}
$$

Performing a similar procedure for the second equation of system (4.2), we have

$$
|\mathrm{y}(\mathrm{t})-\mathrm{n}(\mathrm{t})| \leq \mathrm{M}_{\mathrm{q}} \epsilon_{\mathrm{q}}
$$

Theorem 4.1 Under the hypothesis $\left(\mathrm{H}_{2}\right)$ and if

$$
\Delta=1-\mathrm{N}_{\mathrm{p}} \Omega_{\alpha} \cdot \mathrm{M}_{\mathrm{q}} \Omega_{\chi}>0
$$

holds, then the proposed system (1.1) is stable in the sense of Ulam-Hyers.

Proof Let $(\mathrm{u}, \mathrm{y}) \in \mathrm{E} \times \mathrm{E}$ be the solution of $(2.1)$ and $(\omega, \vartheta) \in \mathrm{E} \times \mathrm{E}$ be the unique solution to the system given by

$$
\begin{cases}D^{p} \omega(t)-\alpha\left(t, \vartheta(t), D^{p} \omega(t)\right)=0 ; & \\ D^{q} \vartheta(t)-\chi\left(t, \omega(t), D^{q} \vartheta(t)\right)=0 ; & \\ \left.D^{p-2} \omega(t)\right|_{t=0^{+}}=\left.\gamma_{1} D^{p-2} \omega(t)\right|_{t=T^{-}}, & \left.D^{p-1} \omega(t)\right|_{t=0^{+}}=\left.\beta_{1} D^{p-1} \omega(t)\right|_{t=T^{-}}, \\ \left.D^{q-2} \vartheta(t)\right|_{t=0^{+}}=\left.\gamma_{2} D^{q-2} \vartheta(t)\right|_{t=T^{-}}, & \left.D^{q-1} \vartheta(t)\right|_{t=0^{+}}=\left.\beta_{2} D^{q-1} \vartheta(t)\right|_{t=T^{-}},\end{cases}
$$

where $t \in J$. Then in view of Lemma 2.1, for $t \in J$, we have the solution of (4.5)

$$
\left\{\begin{array}{l}
\omega(\mathrm{t})=\int_{0}^{\mathrm{T}} \mathrm{G}_{\mathrm{p}}(\mathrm{t}, \mathrm{s}) \alpha\left(\mathrm{s}, \vartheta(\mathrm{s}), \mathrm{D}^{\mathrm{p}} \omega(\mathrm{s})\right) \mathrm{d} \mathrm{s} \\
\vartheta(\mathrm{t})=\int_{0}^{\mathrm{T}} \mathrm{G}_{\mathrm{q}}(\mathrm{t}, \mathrm{s}) \chi\left(\mathrm{s}, \omega(\mathrm{s}), \mathrm{D}^{\mathrm{q}} \vartheta(\mathrm{s})\right) \mathrm{ds} .
\end{array}\right.
$$




\section{Consider}

$$
\begin{aligned}
|\mathrm{u}(\mathrm{t})-\omega(\mathrm{t})| \leq & |\mathrm{u}(\mathrm{t})-\mathrm{m}(\mathrm{t})|+|\mathrm{m}(\mathrm{t})-\omega(\mathrm{t})| \\
\leq & \mathrm{N}_{\mathrm{p}} \epsilon_{\mathrm{p}}+\mid \frac{1}{\Gamma(\mathrm{p})} \int_{0}^{\mathrm{t}}(\mathrm{t}-\mathrm{s})^{\mathrm{p}-1} \mathrm{ds}+\frac{\beta_{1} \mathrm{t}^{\mathrm{p}-1}}{\left(1-\beta_{1}\right) \Gamma(\mathrm{p})} \int_{0}^{\mathrm{T}} \mathrm{ds} \\
& +\frac{\gamma_{1} \mathrm{t}^{\mathrm{p}-2}}{\left(1-\beta_{1}\right)\left(1-\gamma_{1}\right) \Gamma(\mathrm{p}-1)} \int_{0}^{\mathrm{T}}\left[\mathrm{T}-\left(1-\beta_{1}\right) \mathrm{s}\right] \mathrm{d} \mathrm{s}|| \mathrm{v}(\mathrm{s})-\mathrm{v}_{\omega}(\mathrm{s}) \mid,
\end{aligned}
$$

where $\mathrm{v}, \mathrm{v}_{\omega} \in \mathrm{E}$ are of the form

$$
\begin{aligned}
& \mathrm{v}(\mathrm{t})=\alpha(\mathrm{t}, \mathrm{y}(\mathrm{t}), \mathrm{v}(\mathrm{t})), \\
& \mathrm{v}_{\omega}(\mathrm{t})=\alpha\left(\mathrm{t}, \vartheta(\mathrm{t}), \mathrm{v}_{\omega}(\mathrm{t})\right) .
\end{aligned}
$$

By $\left(\mathrm{H}_{2}\right)$, we get

$$
\begin{aligned}
\left|\mathrm{v}(\mathrm{t})-\mathrm{v}_{\omega}(\mathrm{t})\right| & =\left|\alpha(\mathrm{t}, \mathrm{y}(\mathrm{t}), \mathrm{v}(\mathrm{t}))-\alpha\left(\mathrm{t}, \vartheta(\mathrm{t}), \mathrm{v}_{\omega}(\mathrm{t})\right)\right| \\
& \leq \mathrm{K}_{\alpha}|\mathrm{y}(\mathrm{t})-\vartheta(\mathrm{t})|+\mathrm{L}_{\alpha}\left|\mathrm{v}(\mathrm{t})-\mathrm{v}_{\omega}(\mathrm{t})\right| .
\end{aligned}
$$

We obtain

$$
\left|\mathrm{v}(\mathrm{t})-\mathrm{v}_{\omega}(\mathrm{t})\right| \leq \Omega_{\alpha}|\mathrm{y}(\mathrm{t})-\vartheta(\mathrm{t})| .
$$

Using (iii) of (3.2) and (4.7) in (4.6), we get

$$
\|\mathrm{u}-\omega\|_{\mathrm{E}} \leq \mathrm{N}_{\mathrm{p}} \epsilon_{\mathrm{p}}+\mathrm{N}_{\mathrm{p}} \Omega_{\alpha}\|\mathrm{y}-\vartheta\|_{\mathrm{E}}
$$

and similarly we have

$$
\|y-\vartheta\|_{E} \leq M_{q} \epsilon_{q}+M_{q} \Omega_{\chi}\|u-\omega\|_{E},
$$

where $\mathrm{z}, \mathrm{z}_{\vartheta} \in \mathrm{E}$, in the form

$$
\begin{aligned}
& \mathrm{z}(\mathrm{t})=\chi(\mathrm{t}, \mathrm{y}(\mathrm{t}), \mathrm{z}(\mathrm{t})), \\
& \mathrm{z}_{\vartheta}(\mathrm{t})=\chi\left(\mathrm{t}, \omega(\mathrm{t}), \mathrm{z}_{\vartheta}(\mathrm{t})\right) .
\end{aligned}
$$

We write (4.8) and (4.9) as

$$
\begin{aligned}
& \|\mathrm{u}-\omega\|_{\mathrm{E}}-\mathrm{N}_{\mathrm{p}} \Omega_{\alpha}\|\mathrm{y}-\vartheta\|_{\mathrm{E}} \leq \mathrm{N}_{\mathrm{p}} \epsilon_{\mathrm{p}}, \\
& \|\mathrm{y}-\vartheta\|_{\mathrm{E}}-\mathrm{M}_{\mathrm{q}} \Omega_{\chi}\|\mathrm{u}-\omega\|_{\mathrm{E}} \leq \mathrm{M}_{\mathrm{q}} \epsilon_{\mathrm{q}}, \\
& {\left[\begin{array}{cc}
1 & -\mathrm{N}_{\mathrm{p}} \Omega_{\alpha} \\
-\mathrm{M}_{\mathrm{q}} \Omega_{\chi} & 1
\end{array}\right]\left[\begin{array}{l}
\|\mathrm{u}-\omega\|_{\mathrm{E}} \\
\|\mathrm{y}-\vartheta\|_{\mathrm{E}}
\end{array}\right] \leq\left[\begin{array}{l}
\mathrm{N}_{\mathrm{p}} \epsilon_{\mathrm{p}} \\
\mathrm{M}_{\mathrm{q}} \epsilon_{\mathrm{q}}
\end{array}\right] .}
\end{aligned}
$$

Solving the above inequality, we have

$$
\left[\begin{array}{l}
\|u-\omega\|_{E} \\
\|y-\vartheta\|_{E}
\end{array}\right] \leq\left[\begin{array}{cc}
\frac{1}{\Delta} & \frac{\mathrm{N}_{\mathrm{p}} \Omega_{\alpha}}{\Delta} \\
\frac{\mathrm{M}_{\mathrm{q}} \Omega_{\chi}}{\Delta} & \frac{1}{\Delta}
\end{array}\right]\left[\begin{array}{l}
\mathrm{N}_{\mathrm{p}} \epsilon_{\mathrm{p}} \\
\mathrm{M}_{\mathrm{q}} \epsilon_{\mathrm{q}}
\end{array}\right],
$$


where

$$
\Delta=1-\mathrm{N}_{\mathrm{p}} \Omega_{\alpha} \cdot \mathrm{M}_{\mathrm{q}} \Omega_{\chi}>0
$$

Further simplification gives

$$
\begin{aligned}
\|u-\omega\|_{E} & \leq \frac{N_{p} \epsilon_{p}}{\Delta}+\frac{M_{q} N_{p} \Omega_{\alpha} \epsilon_{\mathrm{q}}}{\Delta}, \\
\|y-\vartheta\|_{E} & \leq \frac{M_{q} \epsilon_{q}}{\Delta}+\frac{M_{q} N_{p} \Omega_{\chi} \epsilon_{p}}{\Delta},
\end{aligned}
$$

from which we have

$$
\|\mathrm{u}-\omega\|_{\mathrm{E}}+\|\mathrm{y}-\vartheta\|_{\mathrm{E}} \leq \frac{\mathrm{N}_{\mathrm{p}} \epsilon_{\mathrm{p}}}{\Delta}+\frac{\mathrm{M}_{\mathrm{q}} \epsilon_{\mathrm{q}}}{\Delta}+\frac{\mathrm{M}_{\mathrm{q}} \mathrm{N}_{\mathrm{p}} \Omega_{\alpha} \epsilon_{\mathrm{q}}}{\Delta}+\frac{\mathrm{M}_{\mathrm{q}} \mathrm{N}_{\mathrm{p}} \Omega_{\chi} \epsilon_{\mathrm{p}}}{\Delta} .
$$

Let $\max \left\{\epsilon_{\mathrm{p}}, \epsilon_{\mathrm{q}}\right\}=\epsilon$, then from (4.10) we have

$$
\|(\mathrm{u}, \mathrm{y})-(\omega, \vartheta)\|_{\mathrm{E} \times \mathrm{E}} \leq C_{\mathrm{p}, \mathrm{q}} \epsilon \text {, }
$$

where

$$
C_{p, q}=\left[\frac{\mathrm{N}_{\mathrm{p}}}{\Delta}+\frac{\mathrm{M}_{\mathrm{q}}}{\Delta}+\frac{\mathrm{M}_{\mathrm{q}} \mathrm{N}_{\mathrm{p}} \Omega_{\alpha}}{\Delta}+\frac{\mathrm{M}_{\mathrm{q}} \mathrm{N}_{\mathrm{p}} \Omega_{\chi}}{\Delta}\right] .
$$

Remark 4.1 By setting $\Theta_{\mathrm{p}, \mathrm{q}}(\epsilon)=C_{\mathrm{p}, \mathrm{q}} \epsilon, \Theta_{\mathrm{p}, \mathrm{q}}(0)=0$ in (4.11), then by Definition 2.4 the proposed system (1.1) is generalized Ulam-Hyers stable.

$\left(\mathrm{H}_{4}\right)$ Suppose $\Phi_{\mathrm{p}}, \Phi_{\mathrm{q}} \in\left(J, \mathbb{R}^{+}\right)$are increasing functions. Then there are $\Lambda_{\Phi_{\mathrm{p}}}, \Lambda_{\Phi_{\mathrm{q}}}>0$, such that, for each $t \in J$, the given inequalities

$$
\mathrm{I}^{\mathrm{p}} \Phi_{\mathrm{p}}(\mathrm{t}) \leq \Lambda_{\Phi_{\mathrm{p}}} \Phi_{\mathrm{p}}(\mathrm{t})
$$

and

$$
\mathrm{I}^{\mathrm{q}} \Phi_{\mathrm{q}}(\mathrm{t}) \leq \Lambda_{\Phi_{\mathrm{q}}} \Phi_{\mathrm{q}}(\mathrm{t})
$$

hold.

Remark 4.2 Under the hypothesis $\left(\mathrm{H}_{4}\right)$ and (4.4) and by using Definitions 2.5 and 2.6, one can repeat the process of Lemma 4.1 and Theorem 4.1, system (1.1) will be Ulam-HyersRassias and generalized Ulam-Hyers-Rassias stable.

\section{Example}

\section{Example 5.1}

$$
\begin{cases}D^{\frac{5}{4}} u(t)-\frac{2+|y(t)|+\left|D^{\frac{5}{4}} u(t)\right|}{70 e^{t+12}\left(1+|y(t)|+\left|D^{\frac{5}{4}} u(t)\right|\right)}=0, & \\ D^{\frac{5}{4}} y(t)-\frac{1}{50}(t \cos u(t)-u(t) \sin (t))-\frac{\left|D^{\frac{5}{4}} y(t)\right|}{25+\left|D^{\frac{5}{4}} y(t)\right|}=0, \\ \left.D^{\frac{-3}{4}} u(t)\right|_{t=0^{+}}=\left.\frac{1}{2} D^{\frac{-3}{4}} u(t)\right|_{t=1^{-}}, & \left.D^{\frac{1}{4}} u((t))\right|_{t=0^{+}}=-\left.D^{\frac{1}{4}} u(t)\right|_{t=1^{-}}, \\ \left.D^{\frac{-3}{4}} y(t)\right|_{t=0^{+}}=\left.\frac{1}{2} D^{\frac{-3}{4}} y(t)\right|_{t=1^{-}}, & \left.D^{\frac{1}{4}} y(t)\right|_{t=0^{+}}=-\left.D^{\frac{1}{4}} y(t)\right|_{t=1^{-}},\end{cases}
$$


where $\mathrm{t} \in[0,1]$. From system (5.1), we can see $\mathbf{p}=\mathbf{q}=\frac{5}{4}, \mathrm{~T}=1, \gamma_{1}=\gamma_{2}=\frac{1}{2}$ and $\beta_{1}=\beta_{2}=$ -1 . Also, we can easily find $\mathrm{K}_{\alpha}=\mathrm{L}_{\alpha}=\frac{1}{70 e^{12}}$ and $\mathrm{K}_{\chi}=\mathrm{L}_{\chi}=\frac{1}{25}$. Therefore

$$
\mathrm{N}_{\mathrm{p}} \cdot \Omega_{\alpha}+\mathrm{M}_{\mathrm{q}} \cdot \Omega_{\chi} \approx 0.08847<1
$$

Hence, system (5.1) has a unique solution. Moreover, condition (4.4) also is satisfied. Thus, system (5.1) is Ulam-Hyers stable, generalized Ulam-Hyers stable, Ulam-Hyers-Rassias stable and generalized Ulam-Hyers-Rassias stable.

\section{Conclusion}

We have derived necessary conditions for the existence, uniqueness and different kinds of stability in the sense of Ulam for the solutions of the proposed toppled system (1.1). The required results have been obtained by using classical fixed point theory due to Banach and Leray-Schauder of cone type. Additionally, we have established appropriate conditions for various kinds of Ulam stability to the solutions of the proposed toppled system (1.1). For the justification, we have presented an example which supported the main theoretical results.

\section{Acknowledgements}

Authors would like to thank the referees for suggestions to improve this paper in the current form.

Funding

There is no funding source to support this manuscript financially.

\section{Abbreviations}

FODEs, Fractional order differential equations.

\section{Availability of data and materials}

Not applicable.

\section{Competing interests}

There are no competing interests regarding this research work.

\section{Authors' contributions}

The authors have contributed equally to this manuscript. They read and approved the final manuscript.

\section{Author details}

${ }^{1}$ Department of Mathematics, University of Peshawar, Peshawar, Pakistan. ${ }^{2}$ Department of Mathematics, University of Malakand, Dir(L), Pakistan.

\section{Publisher's Note}

Springer Nature remains neutral with regard to jurisdictional claims in published maps and institutional affiliations.

\section{References}

1. Ahmad, B., Nieto, J.J: Existence of solutions for nonlocal boundary value problems of higher-order nonlinear fractional differential equations. Abstr. Appl. Anal. 2009, Article ID 494720 (2009)

2. Ahmad, B., Nieto, J.J. Existence results for a coupled system of nonlinear fractional differential equations with three-point boundary conditions. Comput. Math. Appl. 58, 1838-1843 (2009)

3. Ahmad, B., Nieto, J.J.: Riemann-Liouville fractional differential equations with fractional boundary conditions. Fixed Point Theory 13(2), 329-336 (2012)

4. Ahmad, N., Ali, Z., Shah, K., Zada, A., Rahman, G.: Analysis of implicit type nonlinear dynamical problem of impulsive fractional differential equations. Complexity 2018, Article ID 6423974 (2018)

5. Ali, A., Rabieib, F., Shah, K.: On Ulam's type stability for a class of impulsive fractional differential equations with nonlinear integral boundary conditions. J. Nonlinear Sci. Appl. 10, 4760-4775 (2017)

6. Ali, A., Samet, B., Shah, K., Khan, R.A.: Existence and stability of solution to a toppled systems of differential equations of non-integer order. Bound. Value Probl. 2017, 16 (2017)

7. Ali, Z., Zada, A., Shah, K.: Existence and stability analysis of three point boundary value problem. Int. J. Appl. Comput. Math. 3(1), 651-664 (2017) 
8. Ali, Z., Zada, A., Shah, K.: Ulam stability results for the solutions of nonlinear implicit fractional order differential equations. Hacet. J. Math. Stat. (2018). https://doi.org/10.15672/HJMS.2018.575

9. Ali, Z., Zada, A., Shah, K.: On Ulam's stability for a coupled systems of nonlinear implicit fractional differential equations. Bull. Malays. Math. Sci. Soc. (2018). https://doi.org/10.1007/s40840-018-0625-x

10. Bai, C., Fang, J.: The existence of a positive solution for a singular coupled system of nonlinear fractional differential equations. Appl. Math. Comput. 150, 611-621 (2004)

11. Benchohra, M., Bouriah, S.: Existence and stability results for nonlinear boundary value problem for implicit differential equations of fractional order. Moroccan J. Pure Appl. Anal. 1(1), 22-37 (2015)

12. Benchohra, M., Bouriah, S., Henderson, J.: Existence and stability results for nonlinear implicit neutral fractional differential equations with finite delay and impulses. Commun. Appl. Nonlinear Anal. 22, 46-67 (2015)

13. Benchohra, M., Hamani, S., Ntouyas, S.K.: Boundary value problems for differential equations with fractional order and nonlocal conditions. Nonlinear Anal. 71, 2391-2396 (2009)

14. Brillouët-Belluot, N., Brzdęk, J., Ciepliński, K.: On some recent developments in Ulam's type stability. Abstr. Appl. Anal. 2012, Article ID 716936 (2012)

15. Chen, Y., An, H.: Numerical solutions of coupled Burgers equations with time and space fractional derivatives. Appl. Math. Comput. 200, 87-95 (2008)

16. Gafiychuk, V., Datsko, B., Meleshko, V., Blackmore, D.: Analysis of the solutions of coupled nonlinear fractional reaction-diffusion equations. Chaos Solitons Fractals 41, 1095-1104 (2009)

17. Gejii, V.D.: Positive solutions of a system of non-autonomous fractional differential equations. J. Math. Anal. Appl. 302 56-64 (2005)

18. Hilfer, R.: Applications of Fractional Calculus in Physics. World Scientific, Singapore (2000)

19. Hyers, D.H.: On the stability of the linear functional equation. Proc. Natl. Acad. Sci. USA 27(4), 222-224 (1941)

20. Hyers, D.H., Isac, G., Rassias, T.M.: Stability of Functional Equations in Several Variables. Birkhäuser, Boston (1998)

21. Jung, S.M.: Hyers-Ulam stability of linear differential equations of first order. Appl. Math. Lett. 19, 854-858 (2006)

22. Khan, A., Shah, K., Li, Y., Khan, T.S.: Ulam type stability for a coupled systems of boundary value problems of nonlinear fractional differential equations. J. Funct. Spaces 2017, Article ID 3046013 (2017)

23. Kilbas, A.A., Srivastava, H.M., Trujillo, J.J.: Theory and Applications of Fractional Differential Equations. North-Holland Mathematics Studies, vol. 204. Elsevier, Amsterdam (2006)

24. Lakshmikantham, V., Leela, S., Devi, J.V.: Theory of Fractional Dynamic Systems. Cambridge Academic Publishers, Cambridge (2009)

25. Lazarević, M.P.: Finite time stability analysis of $\mathrm{PD}^{\alpha}$ fractional control of robotic time-delay systems. Mech. Res. Commun. 33, 269-279 (2006)

26. Li, M., Wang, J., O'Regan, D.: Existence and Ulam's stability for conformable fractional differential equations with constant coefficients. Bull. Malays. Math. Sci. Soc. (2017). https://doi.org/10.1007/s40840-017-0576-7

27. Li, T., Zada, A.: Connections between Hyers-Ulam stability and uniform exponential stability of discrete evolution families of bounded linear operators over Banach spaces. Adv. Differ. Equ. 2016, 153 (2016)

28. Li, T., Zada, A., Faisal, S.: Hyers-Ulam stability of nth order linear differential equations. J. Nonlinear Sci. Appl. 9, 2070-2075 (2016)

29. Liu, F., Burrage, K.: Novel techniques in parameter estimation for fractional dynamical models arising from biological systems. Comput. Math. Appl. 62, 822-833 (2011)

30. Meral, F., Royston, T., Magin, R.: Fractional calculus in viscoelasticity: an experimental study. Commun. Nonlinear Sci. Numer. Simul. 15, 939-945 (2010)

31. Nigmatullin, R., Omay, T., Baleanu, D.: On fractional filtering versus conventional filtering in economics. Commun. Nonlinear Sci. Numer. Simul. 15,979-986 (2010)

32. Oldham, K.: Fractional differential equations in electrochemistry. Adv. Eng. Softw. 41, 9-12 (2010)

33. Podlubny, I.: Fractional Differential Equations. Mathematics in Science and Engineering. Academic Press, New York (1999)

34. Rassias, T.M.: On the stability of functional equations and a problem of Ulam. Acta Appl. Math. 62, 23-130 (2000)

35. Rus, I.A.: Ulam stabilities of ordinary differential equations in a Banach space. Carpath. J. Math. 26, 103-107 (2010)

36. Shah, K., Khalil, H., Khan, R.A.: Investigation of positive solution to a coupled system of impulsive boundary value problems for nonlinear fractional order differential equations. Chaos Solitons Fractals $77,240-246$ (2015)

37. Shah, K., Khan, R.A.: Multiple positive solutions to a coupled systems of nonlinear fractional differential equations SpringerPlus 5, 1116 (2016)

38. Shah, K., Tunç, C.: Existence theory and stability analysis to a system of boundary value problem. J. Taibah Univ. Sci. $11,1330-1342(2017)$

39. Shah, K. Wang, J., Khalil, H., Khan, R.A.: Existence and numerical solutions of a coupled system of integral BVP for fractional differential equations. Adv. Differ. Equ. 2018, 149 (2018)

40. Ulam, S.M.: A Collection of the Mathematical Problems. Interscience, New York (1960)

41. Wang, J., Fečkan, M., Zhou, Y.: Ulam's type stability of impulsive ordinary differential equations. J. Math. Anal. Appl. 395, 258-264 (2012)

42. Wang, J., Fečkan, M., Zhou, Y.: Fractional order differential switched systems with coupled nonlocal initial and impulsive conditions. Bull. Sci. Math. 141, 727-746 (2017)

43. Wang, J., Li, X:: A uniform method to Ulam-Hyers stability for some linear fractional equations. Mediterr. J. Math. 13(2), 625-635 (2016)

44. Wang, J., Lv, L., Zhou, W.: Ulam stability and data dependence for fractional differential equations with Caputo derivative. Electron. J. Qual. Theory Differ. Equ. 2011, 63 (2011)

45. Wang, J., Shah, K., Ali, A.: Existence and Hyers-Ulam stability of fractional nonlinear impulsive switched coupled evolution equations. Math. Methods Appl. Sci. 41, 2392-2402 (2018)

46. Wang, J., Zhou, Y., Fečkan, M.: Nonlinear impulsive problems for fractional differential equations and Ulam stability. Comput. Math. Appl. 64, 3389-3405 (2012)

47. Zada, A., Faisal, S., Li, Y.: On the Hyers-Ulam stability of first order impulsive delay differential equations. J. Funct. Spaces 2016, Article ID 8164978 (2016)

48. Zada, A., Shah, O., Shah, R.: Hyers-Ulam stability of non-autonomous systems in terms of boundedness of Cauchy problems. Appl. Math. Comput. 271, 512-518 (2015) 